\title{
Evanescent light scattering with magnetic colloids
}

\author{
V. Blickle, ${ }^{\text {a) }}$ D. Babič, and C. Bechinger \\ 2. Physikalisches Institut, Universität Stuttgart, Pfaffenwaldring 57, 70550 Stuttgart, Germany
}

(Received 26 April 2005; accepted 8 July 2005; published online 29 August 2005)

\begin{abstract}
We measure magnetic interactions between paramagnetic colloidal beads and an external magnetic field by using total internal reflection microscopy (TIRM). Our results demonstrate that TIRM can be applied to absorptive paramagnetic probe particles and thus extends the range of interaction types which can be addressed with this method. With our setup, we can detect magnetic forces on single superparamagnetic particle ranging from about 10 to $600 \mathrm{fN}$. (C) 2005 American Institute of Physics.
\end{abstract} [DOI: $10.1063 / 1.2035888$ ]

Konstanzer Online-Publikations-System (KOPS)

URL: http://nbn-resolving.de/urn:nbn:de:bsz:352-0-412322

Forces close to surfaces play an important role for, e.g., monolayer adsorption, self-assembly, and wetting phenomena. Aside from understanding the interactions between macroscopic objects, in particular forces on a submicron level between a microscopic probe and a surface are of central interest. During recent years, several techniques aiming at the measurement of such surface forces have been developed with atomic force microscopy being probably the most prominent example. An alternative route in studying interfacial forces is total internal reflection microscopy (TIRM) where the scattered intensity of a freely fluctuating transparent colloidal particle under evanescent illumination is studied. This method which was originally suggested by Prieve et al. ${ }^{1}$ is capable to measure interaction potentials between a probe particle, suspended in a liquid, close to a surface with a force resolution on the order of $5 \mathrm{fN}$. Until now TIRM has been successfully employed for studies of double-layer interactions, van der Waals, entropic, light forces, etc. ${ }^{2-7}$ By functionalization of the bead and the surface, the technique can be applied to study interaction types in biological or chemical systems. ${ }^{8}$

In this letter we demonstrate that TIRM can be also applied to slightly absorbing particles. This allows the use of superparamagnetic colloidal probes, thus extending the range of interaction types accessible to TIRM to magnetic forces. We demonstrate the principle of the method by measuring the magnetic susceptibility of single paramagnetic colloidal beads.

When light is reflected at a solid/fluid interface above the critical angle $\Theta_{c}$, an evanescent wave leaks into the fluid. Its intensity decays exponentially from the interface with a characteristic decay length $\beta^{-1}$ which depends on the incident angle $\Theta$, the wavelength, and the refractive indices at the interface. When a colloidal probe particle is sufficiently close to the surface it will be excited by the evanescent field and scatter the light. In the case of transparent particles, the scattering intensity $I$ of the colloid has been experimentally and theoretically demonstrated to obey $I_{\text {scat }} \propto \exp (-\beta z)$ with $z$ being the particle wall distance. ${ }^{9,10}$ Measuring the scattered light intensity (which fluctuates due to Brownian motion) over time thus provides a sensitive and non intrusive method to determine the particle-wall distance distribution. From this the sphere-wall interaction potential can be derived.

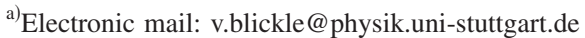

Figure 1 shows a schematic diagram of our setup. The sample cell which consists of two microscope slides separated by a silicone gasket is filled with an aqueous deionized highly diluted colloidal suspension. A laser beam $(\lambda$ $=658.5 \mathrm{~nm}, 13 \mathrm{~mW}$ ) is coupled via a prism into the cell and totally reflected at the glass/water interface. The scattered light is collected using a $(50 \times)$ microscope objective and focused onto a photomultiplier. Lateral positioning of the particle is achieved by two laser tweezers. A Nd:yttriumaluminum-garnet laser $(\lambda=1064 \mathrm{~nm}, 50 \mathrm{~mW})$ is divided into two parts by a polarizing beam splitter. One beam is coupled through a dicroic mirror into the upper microscope objective (upper tweezers). The second beam is focused from below onto the particle, using a $10 \times$ objective (lower tweezers). With a water-cooled electromagnet an external magnetic field $B_{y}$ of up to $300 \mathrm{mT}$ in the $y$ direction can be created. Since the optical setup can be vertically translated with respect to the magnet the magnetic field gradients in the $z$ direction can be precisely adjusted.

As colloidal particles, we used superparamagnetic spheres with diameter $\sigma=2.7$ and $4.5 \mu \mathrm{m}$ (DYNABEADS, Lot.: E467010) which consist of a porous epoxy core with

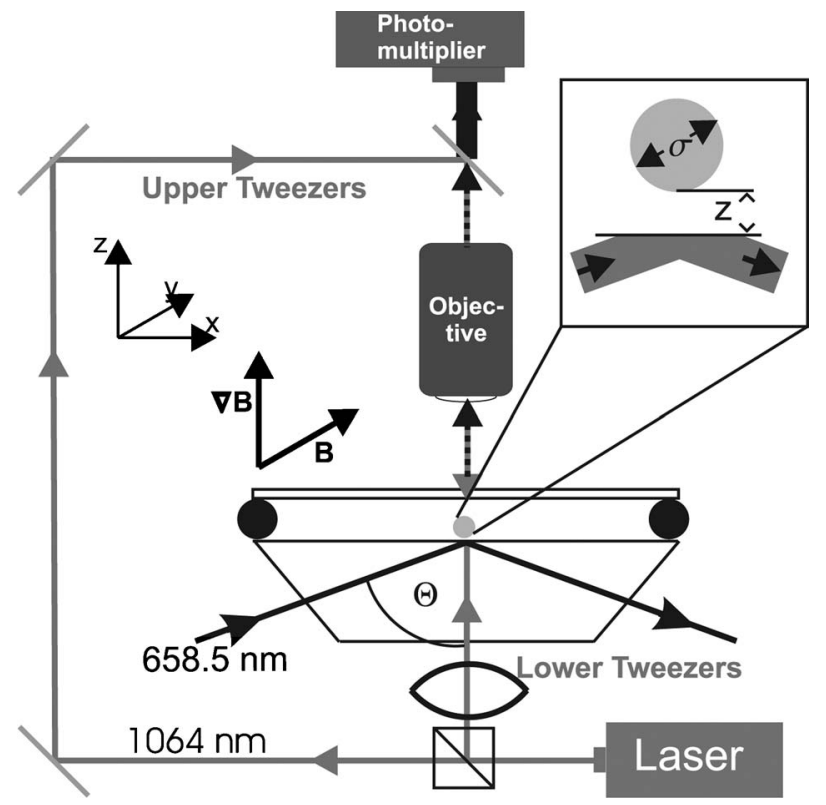

FIG. 1. Schematic diagram of the TIRM setup. The scattered light of the colloidal particle is focused onto a photomultiplier. A magnet creates a magnetic field with adjustable field gradient at the particle position. 
embedded magnetic iron oxide clusters of several tens of nanometers in size. In order to avoid heating effects of the particles due to optical tweezers, their wavelength was chosen to be in the infrared spectrum where absorption of iron oxide is sufficiently small. ${ }^{11}$ In contrast, laser tweezers with $\lambda=532 \mathrm{~nm}$ are partially absorbed in the particles and thus prevent an effective tweezing of superparamagnetic colloids. To avoid irreversible sticking at contact to the surface the particles are additionally sterically stabilized with sodium dodecyle sulfate (SDS). SDS leads also to a negative surface charge of the particles.

So far neither theoretical nor experimental work has been done on TIRM experiments with absorbing particles. Therefore it was not clear a priori weather the exponential distance dependence of $I_{\text {scat }}$ as being valid for transparent colloids, is also justified in case of slightly absorbing particles. When comparing the time dependent scattering intensity of fluctuating superparamagnetic spheres to that of transparent colloids, we realized that in addition to the short time fluctuations of $I_{\text {scat }}$ that are characteristic for translational diffusion, intensity fluctuations on the time scale of several seconds are observed. The latter effect is caused by the rotational diffusion of the superparamagnetic particles which are obviously slightly anisotropic with respect to their scattering properties. In order to suppress rotational diffusion of the particles, which would complicate the analysis of the experiment, we applied either optical tweezers or magnetic fields, which cause the particle axis to be aligned along a preferential direction. As we will show in the following, the assumption of an exponential $\mathrm{z}$ dependence of $I_{\text {scat }}$ leads to potentials which are in agreement with theoretical predictions.

In the presence of optical and magnetic forces, the total interaction energy $W$ of a single particle close to a wall is given by

$$
W=W_{e}+W_{g}+W_{l}+W_{m} .
$$

$W_{e}$ corresponds to electrostatic interaction between the negatively charged particle and the likely charged wall. The distance dependence of this double-layer repulsion is described by a screened electrostatic interaction ${ }^{7} W_{e}=A e^{-\kappa z}$ with the prefactor $A$ given by the surface charges of the particle and the wall and $\kappa$ being the inverse Debye screening length determined by the salt concentration of the suspension. The second term, $W_{g}$ accounts for the graviational force between the particle and the solvent $W_{g}=1 / 3 \pi \sigma^{3}\left(\rho_{p}-\rho_{s}\right) g z$ with $\rho_{p}$ and $\rho_{s}$ being the mass density of particle and solvent and $g$ as the gravitational constant. The last two terms correspond to the optical and magnetic forces whose strength and distance dependence must be experimentally determined as explained in the following.

We first studied the particle wall potentials in the presence of the lower optical tweezers acting on the particle and with the external magnetic field set to zero. As can be seen in Fig. 2(a) the light pressure shifts the potentials away from the wall as the laser power is increased. Having a Rayleigh length of about $5 \mu \mathrm{m}$ we can assume that the light forces do not vary with $z$ for small particle excursions typically encountered in TIRM experiments. Accordingly, the light potential can be written as $W_{l}=F_{l} z$ with $F_{l}$ the optical force given by the optical properties of the particle and the laser intensity. The solid line in Fig. 2(a) shows exemplarily the good agreement between the data and the corresponding fit using Eq. (1). From this we can also determine the screening

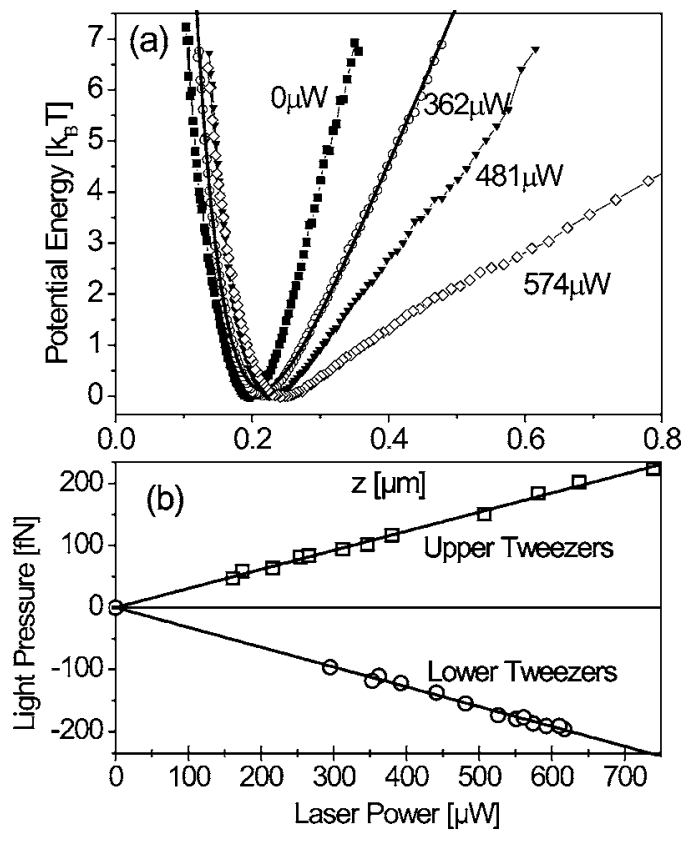

FIG. 2. (a) Interaction potential of a $4.5 \mu \mathrm{m}$ superparamagnetic bead and a glass surface, measured at different laser intensities levels of the lower laser tweezers. (b) Resulting light pressure for upper and lower tweezers.

length to $\kappa^{-1} \simeq 40 \mathrm{~nm}$. Similar curves were also obtained for the upper tweezers. Figure 2(b) shows the laser intensity dependence of the light force for the lower and upper tweezers. It is clearly seen that even in case of slightly absorbing superparamagnetic particles a linear dependence is observed. This strongly suggests that the above assumption of $I_{\text {scat }}$ $\propto \exp (-\beta z)$ is also valid in this case.

In the presence of a magnetic field $B_{y}$, a magnetic dipole moment $M_{y}\left(B_{y}\right)$ is induced in the particles. This leads to a magnetic interaction term $W_{m}=-M_{y} B_{y}$. Being interested on magnetic forces in the $z$ direction we determined the field gradient $\partial B_{y} / \partial z$ along this direction using a hall probe. The field gradient varies linearly with $B_{y}$, i.e., $\partial B_{y} / \partial z$ $=8.66 \mathrm{~m}^{-1} B_{y}$. On the length scale of particles thermal fluctuations $(<1 \mu \mathrm{m})$ the magnetic field $B_{y}$ varies linearly as a function of $z$ so the magnetic interaction $W_{m}$ simplifies to:

$$
W_{m}=-M_{y} \frac{\partial B_{y}}{\partial z} z=F_{m} z
$$

with $F_{m}$ being the magnetic force acting on the particle. Figure 3 shows the resulting potentials for different magnetic

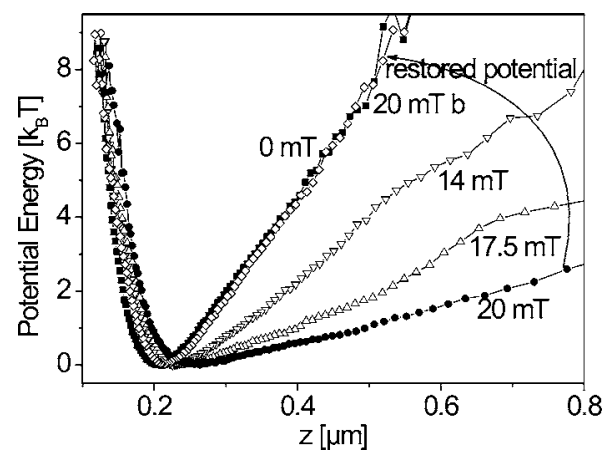

FIG. 3. As Fig. 2 but with the magnetic field varied. At a field of $20 \mathrm{mT}(\mathbf{)}$ the potential is folded back $(\diamond)$ by increasing the power of the upper tweezer. 


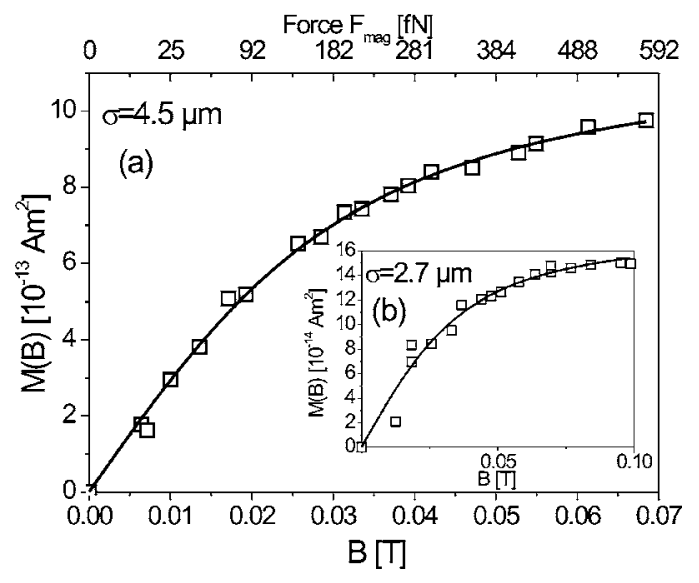

FIG. 4. (a) Magnetisation curve of a $\sigma=4.5 \mu \mathrm{m}$ superparamagnetic particle. The solid line shows a Langevin fit $M(B)=M_{0}[\operatorname{coth}(\alpha B)-1 / \alpha B]$ with parameters: $\alpha=76, M_{0}=1.204 \times 10^{-12} \mathrm{~A} \mathrm{~m}^{2}$. (b) Same curve but for a $\sigma$ $=2.7 \mu \mathrm{m}$ particle. The magnetization and the observed forces are about a factor of 10 smaller.

fields $B_{y}$. In these measurements both tweezers were used to adjust the initial potential. According to Eq. (2) they increase linearly with $z$ for distances larger than $200 \mathrm{~nm}$. Therefore the potential slope difference at large particle/wall distances between potentials measured at finite $B_{y}$ and at $B_{y}=0$ directly yields to $F_{m}$. Using $M_{y}=F_{m}\left(\partial B_{y} / \partial z\right)^{-1}$ [Eq. (2)], $M_{y}$ can be determined as a function of $B_{y}$. With increasing $B_{y}$, however, the linear slope of the potentials would become negative and the particle would not be confined within the evanescent field any more. To avoid such a situation, the optical tweezers can be used to increase the slope of the potential. This is shown by curve $(\diamond)$ in Fig. 3 where the slope of $B_{y}=0$ is restored by increasing the laser power applied with the optical tweezers from above. In this situation $B_{y}$ can be further increased. Iterative application of this procedure allows us to extend the measurements up to $B_{y}=70 \mathrm{mT}$. The resulting $M\left(B_{y}\right)$ curve in Fig. 4 is in perfect agreement with the expected Langevin paramagnetism $M(B)=M_{0}[\operatorname{coth}(\alpha B)-1 / \alpha B]$ with $\alpha=76$ and the saturation magnetisation $M_{0}=1.204 \times 10^{-12} \mathrm{~A} \mathrm{~m}^{2}$. We also performed measurements with $\sigma=2.7 \mu \mathrm{m}$ particles. The corresponding $M(B)$ curve is shown as the inset of Fig. 4.

It should be mentioned that Fig. 4 provides the first single particle measurement of $M(B)$ of superparamagnetic beads. Since such particles are used for magnetic tweezers experiments in biophysical studies, ${ }^{12}$ our results are also useful for the force calibration in such situations. For small magnetic fields $(B<10 \mathrm{mT})$ the magnetic properties of superparamagnetic beads can also be obtained from pair correlation functions on particle ensembles. The obtained parameters agree within a factor of two with our data. ${ }^{13}$ However, since we observed variations of the magnetic properties within one batch of almost $20 \%$, ensemble measurements are quite inapplicable to calibrate single magnetic beads.

In summary, we have demonstrated that TIRM can be also applied to slightly absorptive paramagnetic particles and our results suggest that the assumption of $I_{\text {scat }} \propto \exp (-\beta z)$ is also valid in this case. By appropriate design of the external magnetic field and its gradient, respectively, it should be possible to extend the range of interaction forces accessible with TIRM to the nanoNewton range which is also of interest to interaction measurements in biological systems.

${ }^{1}$ D. Prieve, F. Luo, and F. Lanni, Faraday Discuss. Chem. Soc. 83, 297 (1987).

${ }^{2}$ S. Bike, Curr. Opin. Colloid Interface Sci. 5, 144 (2000).

${ }^{3}$ D. Prieve, Adv. Colloid Interface Sci. 82, 93 (1999).

${ }^{4}$ M. A. Bevan and D. C. Prieve, Langmuir 15, 7925 (1999).

${ }^{5}$ L. Helden, R. Roth, G. H. Koenderink, P. Leiderer, and C. Bechinger, Phys. Rev. Lett. 90, 048301 (2003).

${ }^{6}$ K. Sasaki, M. Tsukima, and H. Masuhara, Appl. Phys. Lett. 71, 37 (1997).

${ }^{7}$ H. H. von Grünberg, L. Helden, P. Leiderer, and C. Bechinger, J. Chem. Phys. 114, 10094 (2001).

${ }^{8}$ S. Robertson and S. Bike, Langmuir 14, 928 (1998).

${ }^{9}$ C. Liu, T. Kaiser, S. Lange, and G. Schweiger, Opt. Commun. 117, 521 (1995).

${ }^{10}$ D. Prieve and J. Walz, Appl. Opt. 32, 1629 (1993).

${ }^{11}$ A. Schlegel, S. Alvarado, and P. Wachter, J. Phys. C 12, 1157 (1979).

${ }^{12}$ A. Bausch, F. Ziemann, A. Boulbitch, K. Jacobson, and E. Sackmann, Biophys. J. 75, 2038 (1998).

${ }^{13} \mathrm{~K}$. Mangold (private communication). 